\title{
Comorbidities of epilepsy: current concepts and future perspectives
}

\author{
Mark R Keezer, Sanjay M Sisodiya, Josemir W Sander
}

The burden of comorbidity in people with epilepsy is high. Several diseases, including depression, anxiety, dementia, migraine, heart disease, peptic ulcers, and arthritis are up to eight times more common in people with epilepsy than in the general population. Several mechanisms explain how epilepsy and comorbidities are associated, including shared risk factors and bidirectional relations. There is a pressing need for new and validated screening instruments and guidelines to help with the early detection and treatment of comorbid conditions. Preliminary evidence suggests that some conditions, such as depression and migraine, negatively affect seizure outcome and quality of life. Further investigation is needed to explore these relations and the effects of targeted interventions. Future advances in the investigation of the comorbidities of epilepsy will strengthen our understanding of epilepsy and could play an important part in stratification for genetic studies.

\section{Introduction}

Young adults have a median of two chronic health conditions, rising to six in people older than 65 years. ${ }^{1}$ These findings have led some researchers to refer to the co-occurrence of several medical conditions in the same individual as a normal state of affairs. ${ }^{2}$ A comorbid condition (or comorbidity) is one that occurs during the course of an index disease (eg, epilepsy). Comorbidities are generally defined in broad terms, including distinct clinical diseases and syndromes, and signs or symptoms of the index disease. ${ }^{1}$

Roughly $50 \%$ of adults with active epilepsy have at least one comorbid medical disorder., ${ }^{1,2}$ Several large population-based studies report various conditions that are up to eight times more prevalent in people with epilepsy relative to the general population. ${ }^{3,4}$ Appreciation of the relevance of these comorbidities is increasing because they affect epilepsy prognosis and quality of life. For example, migraine and psychiatric comorbidities are associated with poor seizure outcome, whereas depression has been linked with reduced quality of life., Despite this growing appreciation, few data are available on the most effective methods to screen for comorbidities and the effect of interventions on prognosis.

Here, we describe ideas relevant to the investigation and conceptualisation of the comorbidities of epilepsy. We review the present state of knowledge of comorbidities associated with epilepsy and explore the ways in which research into comorbidities affords new opportunities for improvement in clinical care and scientific discovery.

\section{Measuring comorbidity}

A fundamental step in the study of the comorbidities of epilepsy is to understand the ways in which comorbidities are measured. An important measure of comorbidity burden is prevalence, which is generally understood to represent point prevalence-the proportion of individuals at risk with the condition in question at a single point in time. ${ }^{7}$ When discussing the comorbidities of epilepsy, the emphasis is on whether the prevalence of comorbid conditions in those with epilepsy is different from that of the general population. ${ }^{8}$ This assessment can be done by comparing prevalence estimates (eg, using $95 \%$ CIs or $\mathrm{p}$ values) or by reporting prevalence ratios. Additional factors, such as sex or age, could have a strong effect on the relative prevalence of two conditions. Adjusted prevalence ratios are an important method to control for such factors and can be calculated using straightforward statistical methods (appendix). ${ }^{9,10}$

Whereas prevalence estimates are used to measure the burden of a single condition, comorbidity indices are useful instruments to measure the overall comorbidity burden of an individual. The Charlson ${ }^{11}$ and the Elixhauser ${ }^{12,13}$ are the most commonly used indices, initially developed for use in observational studies to control for the potentially confounding effect of comorbidity burden on in-hospital mortality, length of hospital stay, and hospitalisation cost. These indices assign points to particular comorbid conditions when present, the sum of which is a weighted score designed to predict prognosis. The Charlson index assigns between 1 and 6 points for 19 different comorbid conditions, whereas the Elixhauser comorbidity measure assigns between -7 and 12 points for 21 conditions. These indices have been well validated in various populations, ${ }^{14}$ but have only recently been validated in people with epilepsy. An epilepsy-specific comorbidity index (ESI) was developed using a population-based administrative database of 7253 individuals with epilepsy in Calgary (AB, Canada)..$^{15}$ The ESI includes 14 conditions (pulmonary circulation disorders, hypertension, cardiac arrhythmias, congestive heart failure, peripheral vascular disease, renal disease, solid tumour without metastases, paraplegia and hemiplegia, aspiration pneumonia, dementia, brain tumour, anoxic brain injury, moderate or severe liver disease, and metastatic cancer), each assigned 1-6 prognostic points. The total ESI score proved to be discriminating, with crude mortality ranging from 4.7 deaths per 1000 person-years for an ESI score of 0 , to 535.6 deaths per 1000 person-years for a score greater than 10. The ESI has been validated and performed well in prospective longitudinal cohorts. ${ }^{16}$
Lancet Neurol 2015 Published Online November 5, 2015 http://dx.doi.org/10.1016 S1474-4422(15)00225-2

National Institute for Health Research University College London Hospitals Biomedical Research Centre, UCL Institute of Neurology, London, UK (M R Keezer MDCM, Prof S M Sisodiya FRCP, Prof JW Sander FR(P); Epilepsy Society, Chalfont St Peter, UK (M R Keezer, S M Sisodiya, JW Sander); and Stichting Epilepsie Instellingen Nederland, Heemstede, Netherlands (J W Sander) Correspondence to: Prof Ley Sander, Box 29, UCL Institute of Neurology, Queen Square, London WC1N 3BG, UK I.sander@ucl.ac.uk See Online for appendix 


\section{Mechanisms of association}

Straightforward measurement of associations between different diseases has been referred to as an "empirical statistical phenomenon that has no meaning in itself". ${ }^{17}$ Measurement of associations is only the first step in a process in which an additional goal is to understand why specific conditions are associated, which in turn might change our understanding of epilepsy and its clinical care.

Several models have been generated to account for the relation between comorbid disorders. ${ }^{17-19}$ Such models are not mutually exclusive and the same comorbid condition could have many reasons for being associated with epilepsy, even in the same individual. Intellectual disability might occur in people with tuberous sclerosis, for example, as a result of the underlying hamartomas or hamartia and as a result of the epileptic seizures (eg, in the context of West syndrome)..$^{20,21}$

A classification scheme for the different mechanisms of association between epilepsy and comorbid conditions has been presented previously. ${ }^{3}$ Here, we build on this initial scheme and incorporate additional aspects, dividing the mechanisms of association into five categories: chance and artifactual comorbidities, causative mechanisms, resultant mechanisms, shared risk factors, and bidirectional effects (figure 1).
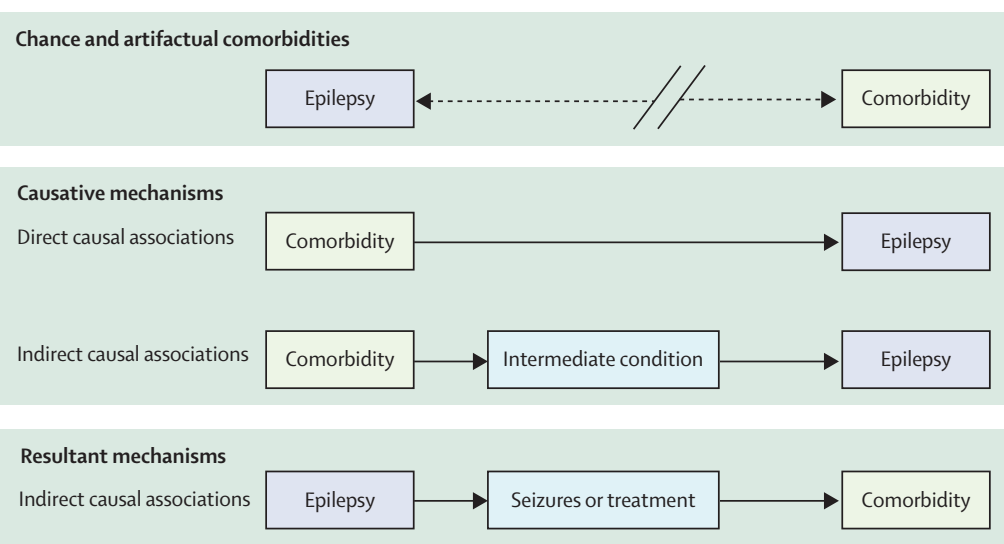

Shared risk factors

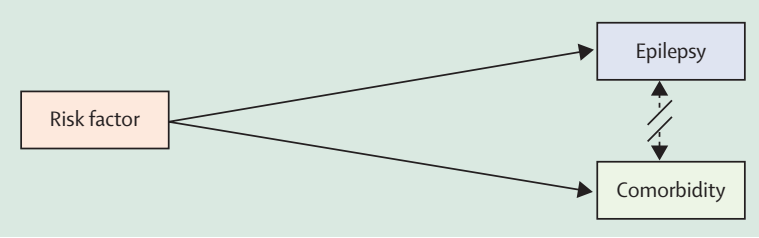

Bidirectional effects

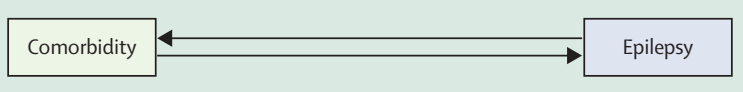

Figure 1: Mechanisms of association between epilepsy and its comorbidities

Each arrow with a solid line represents a casual association, with the cause leading to the effect. Arrows with dashed lines represent non-causal associations.

\section{Chance and artifactual comorbidities}

Chance refers to a circumstance in which the prevalence or incidence of a condition is as frequent in people with epilepsy as would be expected in the general population. Artifactual comorbidity is any non-causal association between epilepsy and a comorbid condition that arises as a result of bias rather than a true causal relation. Artifactual comorbidity can be divided into two major forms: information bias and selection bias.

Information bias is defined as any systematic (ie, nonrandom) error in the data collected that leads to results that are different from the reality. ${ }^{22}$ An apparent rise in the prevalence of a comorbid condition that is only as a result of inaccuracies in the identification of those with epilepsy or the comorbid condition is an example of information bias. Important subtypes of information bias are recall bias (eg, individuals with epilepsy are more likely to recall a past history of mild head trauma than those without epilepsy) and surveillance bias (eg, individuals with epilepsy are more likely to be closely monitored for other diseases). ${ }^{23,24}$

Selection bias, the second form of artifactual comorbidity, occurs when the study population does not accurately represent those individuals initially eligible for inclusion in such a way that systematically alters the relation between conditions. ${ }^{22,25}$ Several types of selection bias are relevant to the investigation of the comorbidities of epilepsy. Referral bias can occur when participants are recruited from a specialised medical setting, in which only people with severe disease are studied, rather than the general population..$^{23}$ Non-response bias arises when particular factors affect some individuals' decisions to participate in a study. ${ }^{25}$ Publication bias is a form of selection bias that is at the level of the overall study rather than the participants, which happens when specific data are not reported, as a result of bias on the part of authors, editors, or peer reviewers. . $^{23,25}$

\section{Causative mechanisms}

Causative mechanisms are one of the simplest mechanisms of association. Here, the comorbid condition arises first, which then gives rise to epilepsy via direct or indirect causal mechanisms. Cerebrovascular disease, for example, directly causes about $10 \%$ of incident epilepsies, ${ }^{26}$ whereas cigarette smoking can indirectly cause epilepsy as a result of neoplasm or stroke.

\section{Resultant mechanisms}

The resultant mechanism of association is similar to the causative model, but the temporal sequence is reversedie, epilepsy takes place first and subsequently gives rise to the comorbid condition. Aspiration pneumonia or seizure-related skeletal fractures are examples of resultant comorbidities of epilepsy. The resultant relation is probably an indirect association caused by the effects of seizures or the associated treatments, although this is yet to be proven. 


\section{Shared risk factors}

Unlike the causative and resultant models, the shared risk factor mechanism of association is not indicative of a causal relation between epilepsy and its comorbidity. This model, in fact, describes a spurious (ie, biased) association as a result of a confounding factor, in which the confounder is defined as a common cause for epilepsy and the comorbid condition. ${ }^{27}$ Unlike the other biased associations as seen in artifactual comorbidity, however, the causal relation between epilepsy, the comorbidity, and the shared risk factor is genuine (figure 1) and its investigation represents both a clinical imperative and a research opportunity. Shared risk factors can be genetic, environmental, structural, or physiological. $^{3}$ Perinatal hypoxaemic brain injury, for example, could result in epilepsy and comorbid spastic paraparesis.

\section{Bidirectional effects}

Bidirectional effects, also known as reciprocal effects, arise when two conditions can each cause the other. Establishing that the temporal sequence is reciprocal (ie, that either could precede the other) is insufficient to prove bidirectionality. Variability in the temporal sequence of epilepsy and a comorbid condition could similarly occur in the context of the shared risk factor model. Some investigators have argued that there is a bidirectional relation between autism spectrum disorder (ASD) and epilepsy. ${ }^{28}$ In the context of tuberous sclerosis, for example, this association is unlikely to be a bidirectional effect; but rather, in some individuals, the ASD might be identified before the onset of epilepsy and in others the reverse is true. This example is evidence of the potentially complex interaction between genetics, structural pathological changes, and environment, rather than evidence that ASD can cause epilepsy and vice versa. In other words, an important distinction exists between bidirectional causality and varying temporal sequence between individuals.

\section{The role of genetics}

The interplay between genetics, epilepsy, and its comorbidities is of interest and warrants particular attention. The various ways in which genetic factors relate to the comorbidities of epilepsy are summarised in figure 2. Perhaps most evident is the way in which genetic mutations might act as shared risk factors-eg, SCN1A mutations predispose individuals to the development of epilepsy and a gait disorder. ${ }^{29}$ Such mutations are examples of genetic pleiotropy, in which the same genes can affect several different traits or disorders. ${ }^{30}$

The interaction between genetics, epilepsy, and its comorbidities extends beyond genetic pleiotropy, however, and could include causative or resultant associations, ${ }^{30}$ in which a genetic factor gives rise to condition A, which in turn causes condition B. A mutation of the TSC1 gene in tuberous sclerosis, for

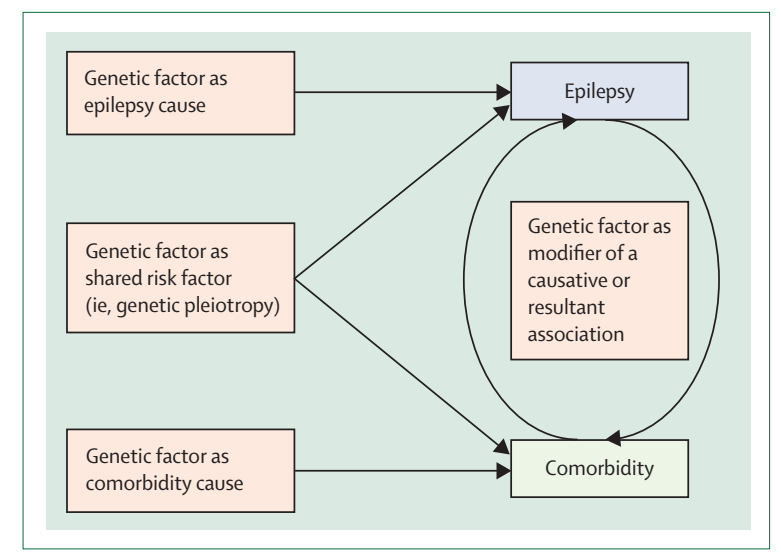

Figure 2: The relation between genetics, epilepsy, and its comorbidities

example, directly results in cortical tubers, some of which (along with surrounding cortical tissue) might result in epilepsy via a causative association. ${ }^{31}$

Genetic factors can also act as modifiers, acting as a third variable that affects the relation between a cause and effect-eg, epilepsy and a comorbidity. ${ }^{32}$ Examples of such relations are the increased risk of epilepsy after traumatic brain injury in carriers of the APOE4 allele, ${ }^{33}$ and the heightened risk of Stevens-Johnson syndrome after the initiation of carbamazepine in carriers of the $H L A B * 1502^{34}$ or $H L A-A * 3101^{35}$ alleles.

\section{The burden of comorbidity}

Several large and comprehensive studies have examined the comorbidity burden in people with epilepsy, and we now describe this research and the relevance of such comorbidities in the context of the concepts underpinning comorbidity research discussed above. These large studies have used population-based cohorts and administrative databases in the $\mathrm{UK}^{36}{ }^{36} \mathrm{Canada},{ }^{37,38}$ and the USA, ${ }^{39-43}$ and have consistently shown a higher prevalence of several somatic and psychiatric conditions in people with epilepsy than in those without epilepsy (table).

Many of the associations between epilepsy and the listed comorbid conditions are not surprising in view of their known roles as causes of epilepsy (eg, CNS neoplasm, stroke, and Alzheimer's disease). ${ }^{44}$ Arrhythmias might occur as a result of the effect of antiepileptic drugs. ${ }^{37}$ Shared risk factors could account for the relation between epilepsy and heart disease (ie, shared vascular risk factors that lead to stroke) or migraine (ie, excessive cortical hyperexcitability). ${ }^{41,45}$ The association between epilepsy and diabetes seems to be especially true for type 1 diabetes and might be related to the shared presence of anti-glutamic acid decarboxylase (GAD) antibodies, which are strongly associated with type 1 diabetes (in about $80 \%$ of individuals) and are present in up to $6 \%$ of people with epilepsy. ${ }^{46}$ Allergies have been suggested to be the effect of specific antiepileptic drugs in some people, and asthma might 
occur as a result of associated environmental and living higher, but their distribution seems to be similar to that conditions that might be indirectly related to epilepsy. ${ }^{3}$ in the general population, with more prevalent The burden of comorbidities in people with epilepsy is migraine, asthma, and brain neoplasms in individuals

\begin{tabular}{|c|c|c|c|c|c|c|c|c|c|}
\hline & $\begin{array}{l}\text { BRFSS (Kobau } \\
\text { et a }\left.\right|^{41} \text { ) }\end{array}$ & $\begin{array}{l}\text { CHIS (Elliott } \\
\text { et } \mathrm{a}^{42} \text { ) }\end{array}$ & 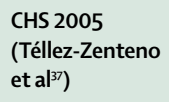 & $\begin{array}{l}\text { CHS } 2007 \\
\text { (Téllez-Zenteno } \\
\text { et a } \mathbf{3}^{38} \text { ) }\end{array}$ & $\begin{array}{l}\text { EPIC (Ottman } \\
\left.\text { et a }\left.\right|^{39}\right)\end{array}$ & $\begin{array}{l}\text { GPRD (Gaitatzis } \\
\text { et a }\left.\right|^{36} \text { ) }\end{array}$ & $\begin{array}{l}\text { NHIS } 2002 \\
\left(\text { Strine et al }\left.\right|^{40}\right)\end{array}$ & $\begin{array}{l}\text { NHIS 2010 } \\
\text { (Kadima } \\
\left.\text { et al }\left.\right|^{43}\right)\end{array}$ & $\begin{array}{l}\text { NPHS } \\
\text { (Téllez-Zenteno } \\
\text { et a } \mathbf{l}^{37} \text { ) }\end{array}$ \\
\hline Source of data (study country) & $\begin{array}{l}\text { Telephone } \\
\text { survey (USA) }\end{array}$ & $\begin{array}{l}\text { Telephone } \\
\text { survey (USA) }\end{array}$ & $\begin{array}{l}\text { Door-to-door } \\
\text { survey (Canada) }\end{array}$ & $\begin{array}{l}\text { Door-to-door } \\
\text { survey (Canada) }\end{array}$ & $\begin{array}{l}\text { Postal survey } \\
\text { (USA) }\end{array}$ & $\begin{array}{l}\text { GP recorded } \\
\text { diagnoses (UK) }\end{array}$ & $\begin{array}{l}\text { Survey with } \\
\text { face-to-face } \\
\text { interviews } \\
\text { (USA) }\end{array}$ & $\begin{array}{l}\text { Survey with } \\
\text { face-to-face } \\
\text { interviews } \\
\text { (USA) }\end{array}$ & $\begin{array}{l}\text { Door-to-door } \\
\text { survey (Canada) }\end{array}$ \\
\hline Reported prevalence & $\begin{array}{l}\%(95 \% \mathrm{Cl}) \text { in } \\
\text { people with } \\
\text { active epilepsy } \\
\text { vs people } \\
\text { without } \\
\text { epilepsy }\end{array}$ & $\begin{array}{l}\%(95 \% \mathrm{Cl}) \text { in } \\
\text { people with vs } \\
\text { people without } \\
\text { epilepsy }\end{array}$ & $\operatorname{PR}(95 \% \mathrm{Cl})$ & $\begin{array}{l}\%(95 \% \mathrm{Cl}) \text { in } \\
\text { people with vs } \\
\text { people without } \\
\text { epilepsy }\end{array}$ & $\mathrm{PR}(95 \% \mathrm{Cl})$ & $\mathrm{PR}(95 \% \mathrm{Cl})$ & $\begin{array}{l}\text { Adjusted }+ \text { PR } \\
(95 \% \mathrm{Cl})\end{array}$ & $\begin{array}{l}\%(95 \% \mathrm{Cl}) \text { in } \\
\text { people with vs } \\
\text { people } \\
\text { without } \\
\text { epilepsy }\end{array}$ & $\mathrm{PR}(95 \% \mathrm{Cl})$ \\
\hline \multicolumn{10}{|l|}{ ICD chapter II: neoplasm } \\
\hline Neoplasm (unspecified) & & $\begin{array}{l}11 \cdot 1 \% \\
(8 \cdot 4-14 \cdot 4) \text { vs } \\
7 \cdot 9 \%(7 \cdot 6-8 \cdot 1)\end{array}$ & $\begin{array}{l}1 \cdot 4 \\
(0 \cdot 9-2 \cdot 1)\end{array}$ & & .. &. & $\begin{array}{l}2 \cdot 4 \\
(1 \cdot 7-3 \cdot 4)\end{array}$ & $\begin{array}{l}11 \cdot 3 \% \\
(8 \cdot 6-14 \cdot 9) \text { vs } \\
8 \cdot 1 \%(7 \cdot 7-8 \cdot 5)\end{array}$ & $\begin{array}{l}1 \cdot 2 \\
(0 \cdot 7-2 \cdot 1)\end{array}$ \\
\hline \multicolumn{10}{|c|}{ ICD chapter IV: endocrine, nutritional, and metabolic diseases } \\
\hline $\begin{array}{l}\text { Diabetes mellitus } \\
\text { (unspecified) }\end{array}$ & $\begin{array}{l}13 \cdot 0 \% \\
(8 \cdot 3-19 \cdot 7) \text { vs } \\
7 \cdot 7 \%(7 \cdot 4-8 \cdot 0)\end{array}$ & $\begin{array}{l}7 \cdot 7 \% \\
(5 \cdot 4-10 \cdot 9) \text { vs } \\
5 \cdot 7 \%(5 \cdot 4-6 \cdot 0) \ddagger\end{array}$ & $\begin{array}{l}1 \cdot 2 \\
(0 \cdot 9-1 \cdot 6)\end{array}$ & .. & $\begin{array}{l}1 \cdot 0 \\
(0 \cdot 9-1 \cdot 1)\end{array}$ & $\begin{array}{l}1 \cdot 6 \\
(1 \cdot 4-1 \cdot 8)\end{array}$ & $\begin{array}{l}1 \cdot 3 \\
(0 \cdot 9-1 \cdot 9)\end{array}$ & $\begin{array}{l}10 \cdot 4 \% \\
(7 \cdot 7-14 \cdot 0) \text { vs } \\
8 \cdot 7 \%(8 \cdot 2-9 \cdot 1)\end{array}$ & $\begin{array}{l}1.6 \\
(1 \cdot 2-2 \cdot 0)\end{array}$ \\
\hline Thyroid condition & .. & .. & $\begin{array}{l}1 \cdot 6 \\
(1 \cdot 3-2 \cdot 1)\end{array}$ & .. & .. & .. & .. & .. & $\begin{array}{l}1 \cdot 3 \\
(0 \cdot 9-1 \cdot 7)\end{array}$ \\
\hline \multicolumn{10}{|c|}{ ICD chapter V: mental and behavioural disorders } \\
\hline Major depression & .. & .. & .. & $\begin{array}{l}17 \cdot 4 \% \\
(10 \cdot 0-24 \cdot 9) \text { vs } \\
10 \cdot 7 \% \\
(10 \cdot 2-11 \cdot 2)\end{array}$ & $\begin{array}{l}1 \cdot 3 \\
(1 \cdot 2-1 \cdot 4)\end{array}$ & $\begin{array}{l}2 \cdot 0 \\
(1 \cdot 9-2 \cdot 1)\end{array}$ &. &.. & .. \\
\hline Anxiety & .. &. & .. & $\begin{array}{l}22 \cdot 8 \% \\
(14 \cdot 8-30 \cdot 9) \text { vs } \\
11 \cdot 2 \% \\
(10 \cdot 8-11 \cdot 7)\end{array}$ & $\begin{array}{l}1 \cdot 6 \\
(1 \cdot 5-1 \cdot 8)\end{array}$ & $\begin{array}{l}2 \cdot 0 \\
(1 \cdot 9-2 \cdot 1)\end{array}$ & .. & .. & .. \\
\hline \multicolumn{10}{|l|}{ ICD chapter VI: nervous system } \\
\hline Dementia (unspecified) & .. &. & .. & .. & .. & $\begin{array}{l}6 \cdot 3 \\
(5 \cdot 5-7 \cdot 4)\end{array}$ &. & .. & $\begin{array}{l}4 \cdot 3 \\
(2 \cdot 7-7 \cdot 4)\end{array}$ \\
\hline Alzheimer's disease & .. & .. & .. & .. & .. & $\begin{array}{l}8 \cdot 1 \\
(5 \cdot 9-11 \cdot 0)\end{array}$ &. &.. & .. \\
\hline Migraine & & & $\begin{array}{l}2 \cdot 0 \\
(1 \cdot 7-2 \cdot 3)\end{array}$ & & $\begin{array}{l}1 \cdot 4 \\
(1 \cdot 3-1 \cdot 5)\end{array}$ & $\begin{array}{l}1 \cdot 6 \\
(1 \cdot 4-1 \cdot 8)\end{array}$ & $\begin{array}{l}3 \cdot 0 \\
(2 \cdot 3-3 \cdot 8) 5\end{array}$ & $\begin{array}{l}34 \cdot 7 \% \\
(30 \cdot 1-39 \cdot 5) \text { vs } \\
16 \cdot 2 \% \\
(15 \cdot 7-16 \cdot 8) \text { ฯ }\end{array}$ & $\begin{array}{l}2 \cdot 6 \\
(2 \cdot 2-3 \cdot 0)\end{array}$ \\
\hline \multicolumn{10}{|l|}{ ICD chapter VII: eye and adnexa } \\
\hline Glaucoma &. &.. & $\begin{array}{l}1 \cdot 2 \\
(0 \cdot 7-1 \cdot 9)\end{array}$ & .. & .. &.. & .. &.. & $\begin{array}{l}1 \cdot 1 \\
(0 \cdot 6-1 \cdot 9)\end{array}$ \\
\hline Cataracts & .. &.. & $\begin{array}{l}1 \cdot 2 \\
(0 \cdot 9-1 \cdot 6)\end{array}$ &.. & .. & .. &.. &.. & $\begin{array}{l}2 \cdot 4 \\
(1 \cdot 9-2 \cdot 9)\end{array}$ \\
\hline \multicolumn{10}{|l|}{ ICD chapter IX: circulatory system } \\
\hline Stroke (unspecified) & $\begin{array}{l}15 \cdot 7 \% \\
(11 \cdot 3-21 \cdot 4) \text { vs } \\
2 \cdot 4 \%(2 \cdot 3-2 \cdot 6)\end{array}$ & $\begin{array}{l}9 \cdot 4 \% \\
(6 \cdot 9-12 \cdot 8) \text { vs } \\
2 \cdot 2 \%(2 \cdot 0-2 \cdot 4)\end{array}$ & $\begin{array}{l}3 \cdot 9 \\
(2 \cdot 7-5 \cdot 3)\end{array}$ & .. & .. & $\begin{array}{l}7 \cdot 0 \\
(6 \cdot 4-7 \cdot 6)\end{array}$ & $\begin{array}{l}7 \cdot 7 \\
(5 \cdot 1-11 \cdot 7)\end{array}$ & $\begin{array}{l}14 \cdot 3 \% \\
(11 \cdot 1-18 \cdot 2) \text { vs } \\
2 \cdot 4 \%(2 \cdot 2-2 \cdot 7)\end{array}$ & $\begin{array}{l}4 \cdot 7 \\
(3 \cdot 4-6 \cdot 2)\end{array}$ \\
\hline Heart disease (unspecified) & $\begin{array}{l}8 \cdot 4 \% \\
(5 \cdot 6-11 \cdot 8) \text { vs } \\
4 \cdot 6 \%(4 \cdot 3-4 \cdot 8)\end{array}$ & $\begin{array}{l}9 \cdot 7 \% \\
(7 \cdot 3-12 \cdot 9) \text { vs } \\
6 \cdot 2 \%(5 \cdot 9-6 \cdot 5)\end{array}$ & $\begin{array}{l}2 \cdot 3 \\
(1 \cdot 9-2 \cdot 7)\end{array}$ & .. & .. & .. & $\begin{array}{l}2 \cdot 5 \\
(1 \cdot 8-3 \cdot 4)\end{array}$ & $\begin{array}{l}18 \cdot 3 \% \\
(14 \cdot 7-22 \cdot 6) \text { vs } \\
11 \cdot 3 \% \\
(10 \cdot 9-11 \cdot 8)\end{array}$ & $\begin{array}{l}1 \cdot 8 \\
(1 \cdot 4-2 \cdot 3)\end{array}$ \\
\hline \multirow[t]{2}{*}{ Hypertension } &.. & $\begin{array}{l}28 \cdot 8 \% \\
(23 \cdot 8-34 \cdot 3) \text { vs } \\
24 \cdot 8 \% \\
(24 \cdot 2-25 \cdot 3)\end{array}$ & $\begin{array}{l}1 \cdot 1 \\
(0 \cdot 9-1 \cdot 3)\end{array}$ & .. & $\begin{array}{l}1 \cdot 0 \\
(0 \cdot 9-1 \cdot 1)\end{array}$ &.. &. & $\begin{array}{l}34 \cdot 2 \% \\
(29 \cdot 7-39 \cdot 0) \text { vs } \\
29 \cdot 0 \% \\
(28 \cdot 4-29 \cdot 6)\end{array}$ & $\begin{array}{l}1 \cdot 9 \\
(1 \cdot 7-2 \cdot 2)\end{array}$ \\
\hline & & & & & & & & \multicolumn{2}{|c|}{ (Table continues on next page) } \\
\hline
\end{tabular}




\begin{tabular}{|c|c|c|c|c|c|c|c|c|c|}
\hline & $\begin{array}{l}\text { BRFSS (Kobau } \\
\text { et } \mathbf{a}^{\left.\right|^{41}} \text { ) }\end{array}$ & $\begin{array}{l}\text { CHIS (Elliott } \\
\text { et } \mathbf{a}^{4^{2}} \text { ) }\end{array}$ & $\begin{array}{l}\text { CHS 2005 } \\
\text { (Téllez-Zenteno } \\
\text { et al }{ }^{\mid 37} \text { ) }\end{array}$ & $\begin{array}{l}\text { CHS } 2007 \\
\text { (Téllez-Zenteno } \\
\text { et a a }{ }^{38} \text { ) }\end{array}$ & $\begin{array}{l}\text { EPIC (Ottman } \\
\left.\text { et } a^{39}\right)\end{array}$ & $\begin{array}{l}\text { GPRD (Gaitatzis } \\
\text { et a }^{\beta^{6}} \text { ) }\end{array}$ & $\begin{array}{l}\text { NHIS } 2002 \\
\left(\text { Strine et } \mathrm{a} \mathrm{l}^{40}\right)\end{array}$ & $\begin{array}{l}\text { NHIS } 2010 \\
\text { (Kadima } \\
\text { et al }\left.\right|^{43} \text { ) }\end{array}$ & $\begin{array}{l}\text { NPHS } \\
\text { (Téllez-Zenteno } \\
\text { et a } \mathbf{a}^{3} \text { ) }\end{array}$ \\
\hline \multicolumn{10}{|c|}{ (Continued from previous page) } \\
\hline \multicolumn{10}{|c|}{ ICD chapter $X$ : respiratory system } \\
\hline COPD &.. & $\begin{array}{l}5 \cdot 2 \% \\
(3 \cdot 6-7 \cdot 6) \text { vs } \\
1 \cdot 9 \%(1 \cdot 8-2 \cdot 1)\end{array}$ & $\begin{array}{l}2 \cdot 9 \\
(2 \cdot 0-4 \cdot 0)\end{array}$ & .. & .. & &. &.. & $\begin{array}{l}1 \cdot 9 \\
(1 \cdot 3-2 \cdot 5)\end{array}$ \\
\hline Chronic bronchitis & .. & .. & .. & .. & .. & $\begin{array}{l}1 \cdot 7 \\
(1 \cdot 3-2 \cdot 2)\end{array}$ &. & $\begin{array}{l}7 \cdot 5 \% \\
(5 \cdot 2-10 \cdot 6) \text { vs } \\
4 \cdot 1 \%(3 \cdot 8-4 \cdot 5)\end{array}$ & .. \\
\hline Emphysema &.. & .. & .. & .. & .. & $\begin{array}{l}1 \cdot 3 \\
(0 \cdot 7-2 \cdot 3)\end{array}$ &. & $\begin{array}{l}5 \cdot 5 \% \\
(3 \cdot 5-8 \cdot 3) \text { vs } \\
1 \cdot 7 \%(1 \cdot 5-2 \cdot 0)\end{array}$ & .. \\
\hline Asthma & $\begin{array}{l}20 \cdot 3 \% \\
(15 \cdot 3-26 \cdot 4) \text { vs } \\
8 \cdot 2 \%(7 \cdot 8-8 \cdot 6)\end{array}$ & $\begin{array}{l}21 \cdot 9 \% \\
(18 \cdot 2-26 \cdot 2) \text { vs } \\
12 \cdot 6 \% \\
(12 \cdot 1-13 \cdot 1)\end{array}$ & $\begin{array}{l}1 \cdot 4 \\
(1 \cdot 1-1 \cdot 7)\end{array}$ & .. & $\begin{array}{l}1 \cdot 3 \\
(1 \cdot 1-1 \cdot 4)\end{array}$ & $\begin{array}{l}1 \cdot 3 \\
(1 \cdot 2-1 \cdot 4)\end{array}$ & $\begin{array}{l}1 \cdot 8 \\
(1 \cdot 4-2 \cdot 4)\end{array}$ & $\begin{array}{l}19 \cdot 2 \% \\
(15 \cdot 2-24 \cdot 0) \text { vs } \\
12 \cdot 6 \% \\
(12 \cdot 0-13 \cdot 2)\end{array}$ & $\begin{array}{l}1 \cdot 1 \\
(0 \cdot 8-1 \cdot 3)\end{array}$ \\
\hline \multicolumn{10}{|l|}{ ICD chapter XI: digestive system } \\
\hline Peptic ulcers & .. & .. & $\begin{array}{l}2 \cdot 5 \\
(2 \cdot 0-3 \cdot 2)\end{array}$ &.. & .. & $\begin{array}{l}1 \cdot 9 \\
(1 \cdot 6-2 \cdot 4)\end{array}$ &.. & $\begin{array}{l}12 \cdot 4 \% \\
(9 \cdot 2-16 \cdot 5) \text { vs } \\
6 \cdot 2 \%(5 \cdot 8-6 \cdot 6)\end{array}$ & $\begin{array}{l}2 \cdot 7 \\
(2 \cdot 1-3 \cdot 4)\end{array}$ \\
\hline $\begin{array}{l}\text { Bowel disorders (Crohn's } \\
\text { and ulcerative colitis) }\end{array}$ &.. &.. & $\begin{array}{l}2 \cdot 0 \\
(1 \cdot 4-2 \cdot 7)\end{array}$ &. & .. &.. &. &.. & $\begin{array}{l}3 \cdot 3 \\
(2 \cdot 4-4 \cdot 3)\end{array}$ \\
\hline \multicolumn{10}{|c|}{ ICD chapter XIII: musculoskeletal system and connective tissues } \\
\hline Allergies &. &. & $\begin{array}{l}1 \cdot 2 \\
(1 \cdot 0-1 \cdot 3)\end{array}$ & .. & .. & .. &. & .. & $\begin{array}{l}1 \cdot 6 \\
(1 \cdot 4-1 \cdot 8)\end{array}$ \\
\hline Back problems & .. &. & $\begin{array}{l}1 \cdot 4 \\
(1 \cdot 2-1 \cdot 6)\end{array}$ & .. & .. &.. &. & .. & $\begin{array}{l}1 \cdot 5 \\
(1 \cdot 3-1 \cdot 7)\end{array}$ \\
\hline Fibromyalgia & .. & .. & $\begin{array}{l}1.5 \\
(0 \cdot 9-2 \cdot 4)\end{array}$ & .. & $\begin{array}{l}2 \cdot 0 \\
(1 \cdot 7-2 \cdot 3)\end{array}$ & .. & .. & .. & .. \\
\hline Arthritis (unspecified) & $\begin{array}{l}43 \cdot 0 \% \\
(37 \cdot 0-49 \cdot 2) \text { vs } \\
28 \cdot 0 \% \\
(27 \cdot 5-28 \cdot 6)\end{array}$ & $\begin{array}{l}32 \cdot 3 \% \\
(27 \cdot 4-37 \cdot 5) \text { vs } \\
18 \cdot 9 \% \\
(18 \cdot 5-19 \cdot 3)\end{array}$ & $\begin{array}{l}1 \cdot 4 \\
(1 \cdot 2-1 \cdot 6)\end{array}$ & .. & .. & .. & $\begin{array}{l}2 \cdot 3 \\
(1 \cdot 7-3 \cdot 0) \|\end{array}$ & $\begin{array}{l}30 \cdot 9 \% \\
(27 \cdot 3-34 \cdot 8) \text { vs } \\
21 \cdot 4 \% \\
(20 \cdot 8-22 \cdot 0)\end{array}$ & $\begin{array}{l}1 \cdot 5 \\
(1 \cdot 3-1 \cdot 7)\end{array}$ \\
\hline \multicolumn{10}{|c|}{ ICD chapter XIV: genitourinary system } \\
\hline Urinary incontinence &.. & .. & $\begin{array}{l}3 \cdot 2 \\
(2 \cdot 4-4 \cdot 1)\end{array}$ & .. & .. & .. & .. & .. & $\begin{array}{l}4 \cdot 4 \\
(3 \cdot 5-5 \cdot 5)\end{array}$ \\
\hline \multicolumn{10}{|c|}{$\begin{array}{l}\text { BRFSS=Behavioural Risk Factor Surveillance System. CHIS=California Health Interview Survey. CHS=Community Health Survey. EPIC=Epilepsy Comorbidities and Health Survey. GPRD=General Practice Research } \\
\text { Database. ICD=International Statistical Classification of Diseases and Related Health Problems. NHIS=National Health Interview Survey. NPHS=National Population Health Survey. GP=general practitioners. } \\
\text { PR=prevalence ratio. OR=prevalence odds ratio. COPD=chronic obstructive pulmonary disease. *Lifetime prevalences are reported, unless otherwise indicated, for comorbidities by ICD-10 chapters; for practical } \\
\text { reasons, the comorbidities listed are those that were recorded in at least two of the studies. †Adjusted for age, sex, race or ethnicity, education, marital, and employment status. fLimited to type } 2 \text { diabetes. } \\
\text { SDefined as a history of severe headache or migraine during the previous } 3 \text { months. } \text {.IIncludes a history of severe headaches. |lIncludes osteoarthritis, rheumatoid arthritis, gout, lupus, and fibromyalgia. }\end{array}$} \\
\hline
\end{tabular}

younger than 64 years and more prevalent cerebrovascular and cardiovascular disease, and meningioma in individuals older than 64 years. ${ }^{36}$

Many studies have consistently reported an increased burden of several comorbidities in people with epilepsy. The degree to which these associations between epilepsy and comorbid conditions result from artifactual comorbidity rather than true causal relations is uncertain. Many of the studies discussed above relied on unvalidated screening instruments to identify individuals with or without epilepsy and with or without comorbidities. ${ }^{36-38,40,42,43}$ Of note, evidence suggests that if individuals with epilepsy are asked whether or not they have epilepsy, around a quarter of the group will say that they do not, ${ }^{47}$ which emphasises the importance of an accurate screening instrument. For conditions such as migraine or fibromyalgia, which are frequently underdiagnosed ${ }^{48,49}$ (especially in the case of mild disease), a higher prevalence in people with epilepsy than in those without epilepsy in these observational and retrospective studies could conceivably be as a result, at least in part, of recall or surveillance bias. The response rate was approximately $50-60 \%$ in several studies, ${ }^{39,41,43}$ rising as high as $75-85 \%$ in others. ${ }^{37,38,40}$ Not surprisingly, investigations that relied on telephone or postal surveys had lower response rates, placing them at particular risk of non-response bias. To maximise accuracy, future studies should be population-based to produce findings that are more generalisable and at lower risk of referral bias, use prospective data collection to reduce the risk of recall bias, 
use only validated screening and diagnostic methods to minimise the risk of misclassification bias while also applying these equally to all participants to minimise surveillance bias, ensure a high response rate to minimise non-response bias, and ensure that all results are reported to minimise the risk of publication bias.

All of the aforementioned studies are cross-sectional (ie, a single snapshot in time) and conclusions regarding whether the comorbid condition or epilepsy arose first are speculative. Some longitudinal studies have been conducted and reported bidirectional effects between epilepsy and several conditions, including depression, ${ }^{50,51}$ suicidality, ${ }^{50,51}$ anxiety, ${ }^{50}$ psychosis or schizophrenia, ${ }^{52,53}$ autism, ${ }^{28}$ migraine, ${ }^{54}$ stroke, ${ }^{55,56}$ and dementia. ${ }^{57,58}$ Whether these associations are truly bidirectional or as a result of variable temporal sequence, however, is unclear. Future studies, preferably experimental studies that can ascertain the causal nature of such associations and the direction of the relation, are needed.

\section{Relevance and implications Screening and diagnosis}

One of the more fundamental features of the comorbidities of epilepsy is their effects on the time of detection of the index disease since they might act as an iatrotropic stimulus. ${ }^{1}$ Regular neurological follow-up in individuals with a known brain neoplasm, for example, will enable earlier detection of a seizure disorder.

Understanding which comorbidities might develop in people with epilepsy is equally relevant. A report by the US Institute of Medicine emphasised the importance of the early identification of comorbid conditions in people with epilepsy. ${ }^{28}$ The idea is that early detection would lead to early intervention and tangible health-care benefits for the patient. Psychiatric, cognitive, and several somatic comorbidities (eg, migraine and osteoporosis) are frequently undetected and undertreated in people with epilepsy. ${ }^{59-62}$ The Institute of Medicine recommended that relevant organisations "establish and disseminate a standard screening protocol for people with epilepsy that implements screening on a regular basis for comorbidities...". ${ }^{28}$ Screening instruments and guidelines exist for conditions such as osteoporosis and depression in the general population; however, these methods have not been translated and validated for use in people with epilepsy. ${ }^{28}$ The absence of such instruments and guidelines is a clear and pressing gap in epilepsy care.

\section{Treatment, prognosis, and quality of life}

The presence of comorbid conditions can affect therapeutic decisions in people with epilepsy. Comorbid hepatic disease or renal insufficiency, migraine, or depression, for example, could be relevant in decisions about antiepileptic drugs, while the risk of cognitive deficits might preclude some surgical options-eg, risk of severe memory impairment from temporal lobectomy.
Investigation of the potential relation between autoimmune disease and epilepsy is expanding. One study reported a statistically significantly elevated prevalence of epilepsy in 135394 individuals with autoimmune disease (ie, type 1 diabetes, psoriasis, rheumatoid arthritis, Graves' disease, Hashimoto's thyroiditis, Crohn's disease, ulcerative colitis, systemic lupus erythematosus, antiphospholipid syndrome, Sjögren's syndrome, myasthenia gravis, and coeliac disease). ${ }^{63}$ This association has potentially important implications for epilepsy treatment in some cases, because, for example, individuals with epilepsy and anti-GAD antibodies can be successfully treated with immunotherapies. ${ }^{64}$

Increasing evidence shows that treating comorbidities might also affect the degree of seizure control. An analysis of the US Food and Drug Administration data on psychotropic drugs, extracted from phase 2 and 3 clinical trials with 75873 participants, showed that those given antidepressants were less likely to have an incident epileptic seizure. ${ }^{65}$ Similarly, treatment of the seizures could affect the comorbidity. The rate of cognitive decline seen with some epilepsies, for example, could be slowed down or even reversed by epilepsy surgery. ${ }^{66}$

Comorbid conditions might allow for so-called prognostic anticipation, ${ }^{1}$ in which their presence could affect, and therefore allow some prediction of, the prognosis of the index disease. Comorbidity has been associated with increased risk of mortality, functional status, quality of life, and different aspects of health care in the context of several index diseases. ${ }^{67}$ For instance, migraine was associated with a reduced probability of early antiepileptic drug response and seizure freedom.? Psychiatric disease was associated with a higher risk of pharmaco-resistance ${ }^{68}$ and worsened outcome after anterior temporal lobectomy. ${ }^{69}$

Comorbid health conditions in children with newonset epilepsy have been associated with reduced quality of life..$^{70}$ Intellectual disabilities and conditions such as depression and attention deficit hyperactivity disorder have been independently associated with poor social adjustment and academic underachievement in children with epilepsy, independent of the severity and type of epilepsy. ${ }^{71-74}$ Similar comorbid conditions in adults, including depression and anxiety, have been associated with reduced quality of life and a higher risk of unemployment. ${ }^{6,75}$

\section{Health-care cost and use}

The health-care costs associated with the co-occurrence of medical conditions in the general population are substantial. An investigation that examined direct healthcare-related costs in a random sample of 1217103 Medicare beneficiaries (aged 65 years or older) in the USA reported that individuals with at least two chronic conditions (defined according to their expected persistence or recurrence) $)^{76}$ represented $65 \%$ of beneficiaries and accounted for $95 \%$ of Medicare expenditures. ${ }^{77}$ An 
examination of American private insurance claims data showed that in people with epilepsy $80 \%$ of direct medical costs were not related to epilepsy, but were related to the treatment of comorbid somatic and psychiatric conditions. $^{78}$ People with epilepsy who have a high comorbidity burden are at increased risk of admission to hospital and generally incur medical costs almost 1.4 times higher than do individuals without such comorbidities. ${ }^{79}$ A study of 824483 American veterans, aged older than 66 years, showed that veterans with newonset epilepsy were at significantly greater risk of medical admission over a 4-year period than were veterans without epilepsy (odds ratio $4 \cdot 84,95 \%$ CI $4 \cdot 29-5 \cdot 46$ ). The five most important predictors of medical admission were heart attack $(4 \cdot 74,2 \cdot 72-8 \cdot 28)$, gallbladder disease $(3 \cdot 90$, $1 \cdot 21-12 \cdot 58)$, anaemia $(2 \cdot 93,2 \cdot 09-4 \cdot 10)$, angina $(2 \cdot 57$, $1 \cdot 57-4 \cdot 22)$, and alcohol dependence $(2 \cdot 46,1 \cdot 49-4 \cdot 08) .{ }^{80}$

\section{Mortality}

Premature mortality in people with epilepsy is increasingly a focus of research. ${ }^{81,82}$ The role of epilepsyrelated causes of death in premature mortality is often emphasised, ${ }^{83}$ but the role of comorbidities should not be overlooked. A meta-analysis of unexpected death in epilepsy showed that only $4 \%$ of deaths in low-risk groups of people with epilepsy in high-income countries were attributable to sudden unexplained death in epilepsy, ${ }^{84}$ which is generally regarded as the most common, single cause of epilepsy-related death. ${ }^{85}$

Almost all deaths in people with epilepsy are related to the comorbidities of epilepsy-particularly neoplasm, cardiovascular, or cerebrovascular disease. ${ }^{86,87}$ Around three-quarters of deaths within one year of epilepsy onset are directly related to the underlying epilepsy cause in individuals with symptomatic epilepsy, rather than seizure-related causes. ${ }^{88}$ Data from the National General Practice Study of Epilepsy, a community-based UK study with almost 25 years of follow-up, has shown that people with epilepsy are more likely than the general population to die of malignant neoplasms, ischaemic heart disease, cerebrovascular disease, and pneumonia, after controlling for the effects of age, sex, and calendar year. ${ }^{89}$ The heightened risk of death from neoplasms persisted even after excluding cerebral neoplasms, and was postulated to be caused by the purported pro-neoplastic effect of some antiepileptic drugs or an underlying shared genetic predisposition. ${ }^{89}$ A report from Sweden showed that individuals with epilepsy and a history of depression or substance abuse were at increased risk of death from external causes, including suicide and accidental death. ${ }^{90}$

\section{Future directions}

Two major ways in which the investigation of comorbidities offers opportunities to further our understanding of epilepsy relate to the evolving notion of epilepsy as a spectrum, or part of a spectrum, and to their use as an important instrument in the study of genetics.
We have generally referred to epilepsy as a single, implicitly uniform entity, but epilepsy is, in fact, highly heterogeneous in terms of its cause, demographics, clinical manifestations, treatment, and prognosis. It is best understood as a collection of individual disorders that share an abnormal tendency to cause epileptic seizures, consisting of dozens of epilepsy syndromes. ${ }^{91}$ Indeed, epilepsy can be rationally defined as a disorder characterised not only by epileptic seizures, but also by its associated biological, psychological, and social conditions. ${ }^{92,93}$ The comorbidities of epilepsy form the core of these associated conditions and contribute to our evolving conceptualisation of epilepsy as a spectrum..$^{94,95}$

Epilepsy can also be regarded as part of a functional spectrum of brain conditions characterised by abnormal paroxysmal neuronal or glial activity. This predisposition towards paroxysmal activity could be deemed a shared risk factor. Some such comorbidities are in the neurological realm, such as migraine, but others, such as depression, fall within psychiatry. The concept of the phenotype of epileptic seizures will not disappear, but an evolving appreciation of the functional range of neurological disease will have a major effect on clinical interpretation, diagnosis, and treatment.

In terms of the genetics of epilepsy, phenotyping is an important technique in the investigation of gene function and the identification of harmful mutations. The process of phenotyping relies on identifying relevant comorbidities in specific groups of individuals. Syndromic epilepsies, with conspicuous congenital comorbidity profiles, lend themselves to genetic investigation; DOORS syndrome, for example, is characterised by a combination of sensorineural deafness, onychodystrophy, osteodystrophy, and intellectual disability with seizures. ${ }^{96}$ One investigation, however, showed that even in individuals with sporadic epilepsy initially thought to be nonsyndromic, genetic factors could account for both the epilepsy and comorbid somatic or psychiatric conditions, some of which might not be evident unless carefully considered or sought. ${ }^{97}$ Irrespective of the potential difficulties, all comorbidities, including even the most inconspicuous, should be regarded as part of the stratification and phenotyping in cases of epilepsy.

\section{Conclusions}

The comorbidities of epilepsy represent a substantial burden for people with epilepsy. The clinical and scientific community should continue to move forward, not only to focus on the description of the statistical relation between different conditions, but also to deconstruct the causal mechanisms for these comorbidities, while bearing in mind the risk of artifactual comorbidity. Screening instruments and guidelines should be developed to help translate the knowledge we have acquired into effective and meaningful clinical interventions. Existing evidence shows that the potential benefit of such endeavours is 


\section{Search strategy and selection criteria}

We searched two electronic databases: Ovid MEDLINE (1946-Nov 25, 2014) and Ovid Embase (1947-Nov 25, 2014) for articles with the MeSH and Emtree terms "comorbidity" and "epilepsy". We additionally searched for titles, abstracts, and keywords using the terms: comorbid*, epilepsy*, seizure $^{*}$, convuls*, and epileptic*. The search was not limited by language of publication. Animal studies were excluded. Our initial search identified 3843 records after the removal of duplicates. The title and abstract of each record were screened by one author (MRK). Additional articles were identified from the authors' own files and from chosen bibliographies. The articles in this Review were included at the authors' discretion on the basis of originality and relevance of the publication.

great. The study of the comorbidities of epilepsy has the potential to transform our understanding of epilepsy, as research into these comorbid conditions helps to clarify the concept of epilepsy as part of a functional spectrum and the important role of genetics.

\section{Contributors}

All authors contributed to the original concept of the manuscript. MRK drafted the manuscript and all authors revised the manuscript.

\section{Declaration of interests}

MRK has received grants and personal fees from UCB Pharma, outside the submitted work. SMS has received research funding from UCB Pharma and personal fees from UCB Pharma, GlaxoSmithKline, and Eisai. JWS has received research funding from Eisai, GlaxoSmithKline, and UCB Pharma, and personal fees from Eisai, UCB Pharma, Lundbeck, and Teva, outside the submitted work. JWS's current position is endowed by the UK Epilepsy Society.

\section{Acknowledgments}

This work was undertaken at the University College London Hospitals NHS Foundation Trust/UCL Comprehensive Biomedical Research Centre, which received a proportion of funding from the UK Department of Health's National Institute for Health Research Biomedical Research Centres funding scheme. JWS receives research support from the Dr Marvin Weil Epilepsy Research Fund and grants from WHO and the Dutch National Epilepsy Fund. The funding sources had no role in study design, data collection, data analysis, data interpretation, writing of the report, or in the decision to submit the manuscript for publication.

\section{References}

1 Feinstein A. Pre-therapeutic classification of co-morbidity in chronic disease. J Chronic Dis 1970; 23: 455-68.

2 Forsgren L. Prevalence of epilepsy in adults in northern Sweden. Epilepsia 1992; 33: 450-58.

3 Gaitatzis A, Sisodiya SM, Sander JW. The somatic comorbidity of epilepsy: a weighty but often unrecognized burden. Epilepsia 2012; 53: 1282-93.

4 LaFrance WC Jr, Kanner AM, Hermann B. Psychiatric comorbidities in epilepsy. Int Rev Neurobiol 2008; 83: 347-83.

5 Velioğlu SK, Boz C, Ozmenoğlu M. The impact of migraine on epilepsy: a prospective prognosis study. Cephalalgia 2005; 25: 528-35.

6 Taylor RS, Sander JW, Taylor RJ, Baker GA. Predictors of health-related quality of life and costs in adults with epilepsy: a systematic review. Epilepsia 2011; 52: 2168-80.

7 Porta M, ed. A Dictionary of Epidemiology. Oxford: Oxford University Press, 2014.

8 Thurman DJ, Beghi E, Begley CE, et al, and the ILAE Commission on Epidemiology. Standards for epidemiologic studies and surveillance of epilepsy. Epilepsia 2011; 52 (suppl 7): 2-26.
9 Deddens JA, Petersen MR. Approaches for estimating prevalence ratios. Occup Environ Med 2008; 65: 501-06.

10 Barros AJ, Hirakata VN. Alternatives for logistic regression in cross-sectional studies: an empirical comparison of models that directly estimate the prevalence ratio. BMC Med Res Methodol 2003; 3: 21.

11 Charlson ME, Pompei P, Ales KL, MacKenzie CR. A new method of classifying prognostic comorbidity in longitudinal studies: development and validation. J Chronic Dis 1987; 40: 373-83.

12 Elixhauser A, Steiner C, Harris DR, Coffey RM. Comorbidity measures for use with administrative data. Med Care 1998; 36: 8-27.

13 van Walraven C, Austin PC, Jennings A, Quan H, Forster AJ. A modification of the Elixhauser comorbidity measures into a point system for hospital death using administrative data. Med Care 2009; 47: 626-33.

14 Sharabiani MT, Aylin P, Bottle A. Systematic review of comorbidity indices for administrative data. Med Care 2012; 50: 1109-18.

15 St Germaine-Smith C, Liu M, Quan H, Wiebe S, Jette N. Development of an epilepsy-specific risk adjustment comorbidity index. Epilepsia 2011; 52: 2161-67.

16 Keezer MR, Bell GS, Jetté N, Sander JW. The performance of three mortality risk-adjustment comorbidity indices in a community epilepsy cohort. Epilepsia 2015; 56: e68-72.

17 Rutter M. Comorbidity: concepts, claims and choices. Crim Behav Ment Health 1997; 7: 265-85.

18 Neale MC, Kendler KS. Models of comorbidity for multifactorial disorders. Am J Hum Genet 1995; 57: 935-53.

19 Rhee SH, Hewitt JK, Lessem JM, Stallings MC, Corley RP, Neale MC. The validity of the Neale and Kendler model-fitting approach in examining the etiology of comorbidity. Behav Genet 2004; 34: 251-65.

20 Jansen FE, Vincken KL, Algra A, et al. Cognitive impairment in tuberous sclerosis complex is a multifactorial condition. Neurology 2008; 70: 916-23.

21 Osborne JP, Lux AL, Edwards SW, et al. The underlying etiology of infantile spasms (West syndrome): information from the United Kingdom Infantile Spasms Study (UKISS) on contemporary causes and their classification. Epilepsia 2010; 51: 2168-74.

22 Rothman KJ, Greenland S, Lash TL. Validity in epidemiologic studies. In: Rothman KJ, Greenland S, Lash TL, eds. Modern epidemiology. 3rd edn. Philadelphia, PA: Lippincott Williams \& Wilkins, 2008: 129-47.

23 Sica GT. Bias in research studies. Radiology 2006; 238: 780-89.

24 Sackett DL. Bias in analytic research. J Chronic Dis 1979; 32: 51-63.

25 Delgado-Rodríguez M, Llorca J. Bias. J Epidemiol Community Health 2004; 58: 635-41.

26 Olafsson E, Ludvigsson P, Gudmundsson G, Hesdorffer D, Kjartansson O, Hauser WA. Incidence of unprovoked seizures and epilepsy in Iceland and assessment of the epilepsy syndrome classification: a prospective study. Lancet Neurol 2005; 4: 627-34

27 Glymour MM, Greenland S. Causal diagrams. In: Rothman KJ, Greenland S, Lash TL, eds. Modern epidemiology. 3rd edn. Philadelphia, PA: Lippincott Williams \& Wilkins, 2008: 183-209.

28 England MJ, Liverman CT, Schultz AM, Strawbridge LM. Epilepsy across the spectrum: promoting health and understanding. A summary of the Institute of Medicine report. Epilepsy Behav 2012; 25: 266-76.

29 Brunklaus A, Ellis R, Reavey E, Forbes GH, Zuberi SM. Prognostic, clinical and demographic features in SCN1A mutation-positive Dravet syndrome. Brain 2012; 135: 2329-36.

30 Ligthart L, Boomsma DI. Causes of comorbidity: pleiotropy or causality? Shared genetic and environmental influences on migraine and neuroticism. Twin Res Hum Genet 2012; 15: 158-65.

31 Major P, Rakowski S, Simon MV, et al. Are cortical tubers epileptogenic? Evidence from electrocorticography. Epilepsia 2009; 50: $147-54$.

32 Greenland S, Rothman KJ, Lash TL. Measures of effect and measures of association. In: Rothman KJ, Greenland S, Lash TL, eds. Modern epidemiology. Philadelphia, PA: Lippincott Williams \& Wilkins, 2008: 51-70.

33 Diaz-Arrastia R, Gong Y, Fair S, et al. Increased risk of late posttraumatic seizures associated with inheritance of APOE epsilon4 allele. Arch Neurol 2003; 60: 818-22. 
34 Tennis P, Stern RS. Risk of serious cutaneous disorders after initiation of use of phenytoin, carbamazepine, or sodium valproate: a record linkage study. Neurology 1997; 49: 542-46.

35 McCormack M, Alfirevic A, Bourgeois S, et al. HLA-A*3101 and carbamazepine-induced hypersensitivity reactions in Europeans. N Engl J Med 2011; 364: 1134-43.

36 Gaitatzis A, Carroll K, Majeed A, Sander JW. The epidemiology of the comorbidity of epilepsy in the general population. Epilepsia 2004; 45: 1613-22.

37 Téllez-Zenteno JF, Matijevic S, Wiebe S. Somatic comorbidity of epilepsy in the general population in Canada. Epilepsia 2005; 46: 1955-62.

38 Téllez-Zenteno JF, Patten SB, Jetté N, Williams J, Wiebe S. Psychiatric comorbidity in epilepsy: a population-based analysis. Epilepsia 2007; 48: 2336-44.

39 Ottman R, Lipton RB, Ettinger AB, et al. Comorbidities of epilepsy: results from the Epilepsy Comorbidities and Health (EPIC) survey. Epilepsia 2011; 52: 308-15.

40 Strine TW, Kobau R, Chapman DP, Thurman DI, Price P, Balluz LS. Psychological distress, comorbidities, and health behaviors among U.S. adults with seizures: results from the 2002 National Health Interview Survey. Epilepsia 2005; 46: 1133-39.

41 Kobau R, Zahran H, Thurman DJ, et al, and the Centers for Disease Control and Prevention (CDC). Epilepsy surveillance among adults-19 states, behavioral risk factor surveillance system, 2005. MMWR Surveill Summ 2008; 57: 1-20.

42 Elliott JO, Lu B, Shneker B, Charyton C, Layne Moore J. Comorbidity, health screening, and quality of life among persons with a history of epilepsy. Epilepsy Behav 2009; 14: 125-29.

43 Kadima N, Kobau R, Zack M, Helmers S, and the Centers for Disease Control and Prevention (CDC). Comorbidity in adults with epilepsyUnited States, 2010. MMWR Morb Mortal Wkly Rep 2013; 62: 849-53.

44 Hauser WA, Annegers JF, Kurland LT. Prevalence of epilepsy in Rochester, Minnesota: 1940-1980. Epilepsia 1991; 32: 429-45.

45 Bauer PR, Carpay JA, Terwindt GM, et al. Headache and epilepsy. Curr Pain Headache Rep 2013; 17: 351.

46 Keezer MR, Novy J, Sander JW. Type 1 diabetes mellitus in people with pharmacoresistant epilepsy: prevalence and clinical characteristics. Epilepsy Res 2015; 115: 55-57.

47 Ottman R, Barker-Cummings C, Leibson CL, Vasoli VM, Hauser WA, Buchhalter JR. Validation of a brief screening instrument for the ascertainment of epilepsy. Epilepsia 2010; 51: 191-97.

48 Cevoli S, D'Amico D, Martelletti P, et al. Underdiagnosis and undertreatment of migraine in Italy: a survey of patients attending for the first time 10 headache centres. Cephalalgia 2009; 29: 1285-93.

49 Di Franco M, Iannuccelli C, Bazzichi L, et al. Misdiagnosis in fibromyalgia: a multicentre study. Clin Exp Rheumatol 2011; 29 (suppl 69): S104-08

50 Hesdorffer DC, Ishihara L, Mynepalli L, Webb DJ, Weil J, Hauser WA. Epilepsy, suicidality, and psychiatric disorders: a bidirectional association. Ann Neurol 2012; 72: 184-91.

51 Hesdorffer DC, Hauser WA, Olafsson E, Ludvigsson P, Kjartansson $\mathrm{O}$. Depression and suicide attempt as risk factors for incident unprovoked seizures. Ann Neurol 2006; 59: 35-41.

52 Qin P, Xu H, Laursen TM, Vestergaard M, Mortensen PB. Risk for schizophrenia and schizophrenia-like psychosis among patients with epilepsy: population based cohort study. BMJ 2005; 331: 23 .

53 Chang YT, Chen PC, Tsai IJ, et al. Bidirectional relation between schizophrenia and epilepsy: a population-based retrospective cohor study. Epilepsia 2011; 52: 2036-42.

54 Ottman R, Lipton RB. Comorbidity of migraine and epilepsy. Neurology 1994; 44: 2105-10.

55 Cleary P, Shorvon S, Tallis R. Late-onset seizures as a predictor of subsequent stroke. Lancet 2004; 363: 1184-86.

56 Kotila M, Waltimo O. Epilepsy after stroke. Epilepsia 1992; 33: 495-98.

57 Hesdorffer DC, Hauser WA, Annegers JF, Kokmen E, Rocca WA. Dementia and adult-onset unprovoked seizures. Neurology 1996; 46: 727-30.

58 Breteler MM, de Groot RR, van Romunde LK, Hofman A. Risk of dementia in patients with Parkinson's disease, epilepsy, and severe head trauma: a register-based follow-up study. Am J Epidemiol 1995; 142: $1300-05$
59 Ott D, Siddarth P, Gurbani S, et al. Behavioral disorders in pediatric epilepsy: unmet psychiatric need. Epilepsia 2003; 44: 591-97.

60 Barry JJ. The recognition and management of mood disorders as a comorbidity of epilepsy. Epilepsia 2003; 44 (suppl 4): 30-40.

61 Kwan P, Man CB, Leung H, Yu E, Wong KS. Headache in patients with epilepsy: a prospective incidence study. Epilepsia 2008; 49: 1099-102.

62 Lado F, Spiegel R, Masur JH, Boro A, Haut SR. Value of routine screening for bone demineralization in an urban population of patients with epilepsy. Epilepsy Res 2008; 78: 155-60.

63 Ong MS, Kohane IS, Cai T, Gorman MP, Mandl KD. Population-leve evidence for an autoimmune etiology of epilepsy. JAMA Neurol 2014; 71: $569-74$

64 Vincent A, Bien CG, Irani SR, Waters P. Autoantibodies associated with diseases of the CNS: new developments and future challenges. Lancet Neurol 2011; 10: 759-72.

65 Alper K, Schwartz KA, Kolts RL, Khan A. Seizure incidence in psychopharmacological clinical trials: an analysis of Food and Drug Administration (FDA) summary basis of approval reports. Biol Psychiatry 2007; 62: 345-54.

66 Téllez-Zenteno JF, Dhar R, Hernandez-Ronquillo L, Wiebe S. Long-term outcomes in epilepsy surgery: antiepileptic drugs, mortality, cognitive and psychosocial aspects. Brain 2007; 130: 334-45.

67 Gijsen R, Hoeymans N, Schellevis FG, Ruwaard D, Satariano WA van den Bos GA. Causes and consequences of comorbidity: a review. J Clin Epidemiol 2001; 54: 661-74.

68 Hitiris N, Mohanraj R, Norrie J, Sills GJ, Brodie MJ. Predictors of pharmacoresistant epilepsy. Epilepsy Res 2007; 75: 192-96.

69 Kanner AM, Byrne R, Chicharro A, Wuu J, Frey M. A lifetime psychiatric history predicts a worse seizure outcome following temporal lobectomy. Neurology 2009; 72: 793-99.

70 Taylor J, Jacoby A, Baker GA, Marson AG. Self-reported and parent-reported quality of life of children and adolescents with new-onset epilepsy. Epilepsia 2011; 52: 1489-98.

71 Dunn DW, Johnson CS, Perkins SM, et al. Academic problems in children with seizures: relationships with neuropsychological functioning and family variables during the 3 years after onset. Epilepsy Behav 2010; 19: 455-61.

72 Kokkonen J, Kokkonen ER, Saukkonen AL, Pennanen P. Psychosocial outcome of young adults with epilepsy in childhood. J Neurol Neurosurg Psychiatry 1997; 62: 265-68.

73 Fastenau PS, Shen J, Dunn DW, Perkins SM, Hermann BP, Austin JK. Neuropsychological predictors of academic underachievement in pediatric epilepsy: moderating roles of demographic, seizure, and psychosocial variables. Epilepsia 2004; 45: 1261-72.

74 Sturniolo MG, Galletti F. Idiopathic epilepsy and school achievement. Arch Dis Child 1994; 70: 424-28.

75 Smeets VM, van Lierop BA, Vanhoutvin JP, Aldenkamp AP, Nijhuis FJ. Epilepsy and employment: literature review. Epilepsy Behav 2007; 10: 354-62.

76 Starfield B, Weiner J, Mumford L, Steinwachs D. Ambulatory care groups: a categorization of diagnoses for research and management. Health Serv Res 1991; 26: 53-74.

77 Wolff JL, Starfield B, Anderson G. Prevalence, expenditures, and complications of multiple chronic conditions in the elderly. Arch Intern Med 2002; 162: 2269-76.

78 Ivanova JI, Birnbaum HG, Kidolezi Y, Qiu Y, Mallett D, Caleo S. Economic burden of epilepsy among the privately insured in the US. Pharmacoeconomics 2010; 28: 675-85.

79 Lee WC, Arcona S, Thomas SK, Wang Q, Hoffmann MS Pashos CL. Effect of comorbidities on medical care use and cost among refractory patients with partial seizure disorder. Epilepsy Behav 2005; 7: 123-26.

80 Copeland LA, Ettinger AB, Zeber JE, Gonzalez JM, Pugh MJ. Psychiatric and medical admissions observed among elderly patients with new-onset epilepsy. BMC Health Serv Res 2011; 11: 84.

81 Jetté N, Wiebe S. Mortality in epilepsy. In: Panayiotopoulos CP, ed. Atlas of epilepsies. London: Springer-Verlag London Limited, 2010: 1353-57.

82 Nevalainen O, Ansakorpi H, Simola M, et al. Epilepsy-related clinical characteristics and mortality: a systematic review and meta-analysis. Neurology 2014; 83: 1968-77. 
83 Moshé SL, Perucca E, Ryvlin P, Tomson T. Epilepsy: new advances. Lancet 2015; 385: 884-98.

84 Téllez-Zenteno JF, Ronquillo LH, Wiebe S. Sudden unexpected death in epilepsy: evidence-based analysis of incidence and risk factors. Epilepsy Res 2005; 65: 101-15.

85 Surges R, Sander JW. Sudden unexpected death in epilepsy: mechanisms, prevalence, and prevention. Curr Opin Neurol 2012; 25: 201-07.

86 Nevalainen O, Raitanen J, Ansakorpi H, Artama M, Isojärvi J, Auvinen A. Long-term mortality risk by cause of death in newly diagnosed patients with epilepsy in Finland: a nationwide registerbased study. Eur J Epidemiol 2013; 28: 981-90.

87 Forsgren L, Hauser WA, Olafsson E, Sander JW, Sillanpää M, Tomson T. Mortality of epilepsy in developed countries: a review. Epilepsia 2005; 46 (suppl 11): 18-27.

88 Loiseau J, Picot MC, Loiseau P. Short-term mortality after a first epileptic seizure: a population-based study. Epilepsia 1999; 40: $1388-92$.

89 Neligan A, Bell GS, Johnson AL, Goodridge DM, Shorvon SD, Sander JW. The long-term risk of premature mortality in people with epilepsy. Brain 2011; 134: 388-95.

90 Fazel S, Wolf A, Långström N, Newton CR, Lichtenstein P. Premature mortality in epilepsy and the role of psychiatric comorbidity: a total population study. Lancet 2013; 382: 1646-54
91 Engel J Jr. Report of the ILAE classification core group. Epilepsia 2006; 47: 1558-68.

92 Fisher RS, van Emde Boas W, Blume W, et al. Epileptic seizures and epilepsy: definitions proposed by the International League Against Epilepsy (ILAE) and the International Bureau for Epilepsy (IBE). Epilepsia 2005; 46: 470-72.

93 Fisher RS. Commentary: operational definition of epilepsy survey. Epilepsia 2014; 55: 1688.

94 Berg AT. Epilepsy, cognition, and behavior: The clinical picture. Epilepsia 2011; 52 (suppl 1): 7-12.

95 Institute of Medicine. Epilepsy across the spectrum: promoting health and understanding. Washington, DC: The National Academies Press, 2012.

96 Campeau PM, Hennekam RC, and the DOORS syndrome collaborative group. DOORS syndrome: phenotype, genotype and comparison with Coffin-Siris syndrome. Am J Med Genet C Semin Med Genet 2014; 166C: 327-32.

97 Kasperavičiūtè D, Catarino CB, Chinthapalli K, et al. Uncovering genomic causes of co-morbidity in epilepsy: gene-driven phenotypic characterization of rare microdeletions. PLoS One 2011; 6: e23182. 